VoL. 43 (1991) [63-71]

\title{
NONSINGULAR RETRACTABLE MODULES AND THEIR ENDOMORPHISM RINGS
}

\author{
SoUmaYa MAKDISSI KhURI
}

\begin{abstract}
A module ${ }_{R} M$ is said to be retractable if $\operatorname{Hom}_{R}(M, U) \neq 0$ for each nonzero submodule $U$ of $M . M$ is said to be a $C S$ module if every complement submodule of $M$ is a direct summand in $M$. Retractable modules are compared to nondegenerate modules on the one hand and to e-retractable modules on the other (nondegenerate implies retractable implies e-retractable); and it is shown that if $M$ is nonsingular and retractable, then $\operatorname{End}_{R} M$ is a left $C S$ ring if and only if $M$ is a $C S$ module.
\end{abstract}

\section{INTRODUCTION AND PRELIMINARIES}

Let ${ }_{R} M$ be a left module over the associative ring $R$ with identity. $M$ is said to be retractable if $\operatorname{Hom}_{R}(M, U) \neq 0$ for every nonzero submodule $U$ of $M$, and e-retractable if $\operatorname{Hom}_{R}(M, C) \neq 0$ for every nonzero complement submodule $C$ of $M$. Let $M *=\operatorname{Hom}_{R}(M, R)$ and let $T=\sum_{f \in M^{*}} \operatorname{Im} f$ be the trace of $M$ in $R ; M$ is said to be nondegenerate if $T m \neq 0$ for every nonzero $m \in M . M$ is said to be a $C S$ module if every complement submodule of $M$ is a direct summand in $M$; a ring $B$ is a left $C S$ ring if ${ }_{B} B$ is a $C S$ module.

Any nondegenerate module is retractable [6, Proposition 3.2], but not conversely (for example, let $M$ be the $Z$-module $Z / p^{n} Z$ ); and any retractable module is clearly $e$-retractable, but not conversely (see for example [5, Example 3.4]). In this paper, we shall be mainly concerned with investigating some properties of a nonsingular ${ }_{R} M$ when it is nondegenerate, retractable or e-retractable, and with the connection between $M$ 's being a CS module and its endomorphism ring $\operatorname{End}_{R} M$ 's being a left $C S$ ring. From [2] and [6] we have the following:

Let ${ }_{R} M$ be nonsingular and nondegenerate; then $\operatorname{End}_{R} M$ is a left $C S$ ring if and only if $M$ is a $C S$ module [6, Corollary 3.11].

Let ${ }_{R} M$ be nonsingular and $e$-retractable ; then, if $\operatorname{End}_{R} M$ is a left $C S$ ring, $M$ is a $C S$ module [2, Theorem 3.1].

Moreover, we know that a nonsingular, e-retractable $M$ can be $C S$ and still have an endomorphism ring which is not left $C S$ (see for example [2, Example 3.3]). Hence,

Received 3 February 1990

Copyright Clearance Centre, Inc. Serial-fee code: 0004-9729/91 \$A2.00+0.00. 
we know that we cannot hope to show that $\operatorname{End}_{R} M$ is left $C S$ if and only if $M$ is $C S$, for a nonsingular e-retractable $M$. However, it is natural to ask whether this conclusion can be generalised from $M$ nonsingular nondegenerate to $M$ nonsingular retractable, and in our main result (Theorem 3.2) we give an affirmative answer to this question. Before proving Theorem 3.2, we take a closer look, in Section 2, at nondegenerate, retractable and $e$-retractable modules and try to bring out the differences between them. We find that these differences stand out most clearly in the ability of each of these types of modules to "preserve essentiality", that is to have " ${ }_{R} U$ is essential in $V$ " imply " $\operatorname{Hom}_{R}(M, U)$ is essential in $\operatorname{Hom}_{R}(M, V)$ " or conversely.

Throughout this paper, let $R$ be an associative ring with identity, ${ }_{R} M$ a left $R$ module and $B=\operatorname{End}_{R} M$ the ring of $R$-endomorphisms of $M . U<{ }_{R} M$ (respectively $U<{ }_{B} B$ ) will mean that $U$ is a submodule of $M$ (respectively a left ideal of $B$ ). $U<{ }_{R}^{\prime} V$ (or simply $U<^{\prime} V$ when the context is clear) will mean that $U$ is essential as an $R$-submodule of $V$, that is $U$ has nonzero intersection with every nonzero $R$ submodule of $V$; and similarly for $H<{ }_{B}^{\prime} B$.

The following well-known property of essential submodules will be used without comment in the sequel:

If $U<{ }_{R} V<{ }_{R} W$, then $U<{ }_{R}^{\prime} W$ if and only if $U<{ }_{R}^{\prime} V$ and $V<{ }_{R}^{\prime} W$.

Let $I_{B}(U)=\{b \in B: M b \subseteq U\}$, for $U<{ }_{R} M$, and let $S_{M}(H)=M B=\sum_{h \in H} M h$, for $H<{ }_{B} B$.

Clearly, $I_{B}(U)$ is a left ideal of $B$ and $S_{M}(H)=M H$ is a submodule of $M$. The notations $S_{M}(H)$ and $M H$ will be used interchangeably, and we will identify $I_{B}(U)$ and $\operatorname{Hom}_{R}(M, U)$ for $U<{ }_{R} M$; in particular, $M$ is retractable if $I_{B}(U) \neq 0$ for $0 \neq U<{ }_{R} M$.

The following Proposition is easily verified:

Proposition 1.1.

(i) $U_{1}<{ }_{R} U_{2}<{ }_{R} M \Rightarrow I_{B}\left(U_{1}\right)<I_{B}\left(U_{2}\right)$.

(ii) $H_{1}<{ }_{B} H_{2}<{ }_{B} B \Rightarrow S_{M}\left(H_{1}\right)<S_{M}\left(H_{2}\right)$.

(iii) $S_{M} I_{B}(U)<U$ and $H<I_{B} S_{M}(H)$, for $U<{ }_{R} M$ and $H<{ }_{B} B$.

(iv) $I_{B}(U)=I_{B} S_{M} I_{B}(U)$ and $S_{M}(H)=S_{M} I_{B} S_{M}(H)$, for $U<{ }_{R} M$ and $H<B B$.

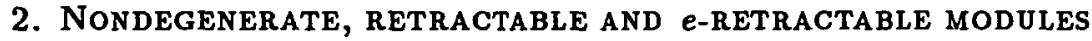

In [6, Proposition 3.2], it was shown that, when $M$ is nondegenerate then $M$ is retractable and $M$ has the following two properties:

(I) For $U<{ }_{R} V<{ }_{R} M, U<{ }_{R}^{\prime} V$ if and only if $I_{B}(U)<I_{B}(V)$.

(II) For $B<{ }_{B} J<{ }_{B} B, H<_{B} J$ if and only if $S_{M}(H)<^{\prime} S_{M}(J)$. 
For retractable modules, we have the following:

PROPOSITION 2.1. For any ${ }_{R} M$, the following are equivalent:

(i) ${ }_{R} M$ is retractable.

(ii) For any $U<{ }_{R} M, S_{M} I_{B}(U)<{ }_{R} U$.

(iii) For $U<{ }_{R} V<{ }_{R} M, I_{B}(U)<I_{B}(V) \Rightarrow U<{ }_{R}^{\prime} V$.

Proof: (i) $\Rightarrow$ (ii): If $M$ is retractable and $0 \neq U<{ }_{R} M$, then, for any $0 \neq u \in U$, there is $0 \neq b \in I_{B}(R u)$; hence, since $I_{B}(R u) \subseteq I_{B}(U)$, we have:

$0 \neq M b \subseteq R u \cap S_{M} I_{B}(U)$, so that $S_{M} I_{B}(U)<{ }_{R}^{\prime} U$.

(ii) $\Rightarrow$ (i): If $S_{M} I_{B}(U)<{ }_{R}^{\prime} U$ for any $U$, then, for $U \neq 0$ this implies $I_{B}(U) \neq 0$.

(i) $\Rightarrow$ (iii): If $M$ is retractable and $I_{B}(U)<^{\prime} I_{B}(V)$ for $U<{ }_{R} V<{ }_{R} M$, then, for any $0 \neq v \in V$, there is $0 \neq b \in I_{B}(R v) \cap I_{B}(U)$; we have: $0 \neq M b \subseteq R v \cap U$, which shows that $U<{ }_{R}^{\prime} V$.

(iii) $\Rightarrow$ (ii): Assume that (iii) holds and let $0 \neq U<{ }_{R} M$. Then, since $I_{B}(U)=$ $I_{B} S_{M} I_{B}(U)$ implies, in particular, that $I_{B} S_{M} I_{B}(U)<I_{B}(U)$, we have, by (iii), that $S_{M} I_{B}(U){ }_{R}^{\prime} U$.

We can see from Proposition 2.1 that whereas for nondegenerate modules we have property (I), for retractable modules we have only one direction of (I). However, if $M$ is nonsingular as well as retractable, then we do have the other direction also.

Recall that ${ }_{R} M$ is nonsingular if, for any $m \in M, \operatorname{Im}=0$ and $I<_{R}^{\prime} R \Rightarrow m=0$.

THEOREM 2.2. If ${ }_{R} M$ is nonsingular and retractable then we have:

(I) For $U<{ }_{R} V<{ }_{R} M, U<{ }_{R}^{\prime} V$ if and only if $I_{B}(U)<I_{B}(V)$.

Proof: By Proposition 2.1, we only need to prove one direction. Assume that $U<{ }_{R} V$ and let $0 \neq b \in I_{B}(V)$. Choose $m \in M$ such that $0 \neq m b=v \in V$; then, since $U<{ }_{R}^{\prime} V$, there is $r \in R$, such that $0 \neq u=r v=r m b$; note here that $0 \neq R r m b=R u \subseteq U$. By hypothesis, we have $S_{M} I_{B}(R r m)<R r m ;$ if $x$ is any nonzero element in $R r m$, then it is known (see for example [3], p.46, Lemma 3) that the left ideal $J_{x}=\left\{r \in R: r x \in S_{M} I_{B}(R r m)\right\}$ is an essential left ideal of $R$.

If $\left[S_{M} I_{B}(R r m)\right] b=0$, then, for any nonzero $x$ in $R r m$, since $J_{x} \subseteq S_{M} I_{B}(R r m)$, we will have $J_{x} x b=0$ and consequently $x b=0$ since $M$ is nonsingular and $J_{x}<{ }_{R}^{\prime} R$; but this contradicts the fact that $R r m b \neq 0$. Hence $\left[S_{M} I_{B}(R r m)\right] b \neq 0$ and there is $c \in I_{B}(R r m)$ such that $c b \neq 0$. Then $0 \neq c b \in B b \cap I_{B}(U)$, proving that $I_{B}(U)<$ $I_{B}(V)$.

A combination of Theorem 2.2 and Proposition 2.1 gives:

Corollary 2.3. Let ${ }_{R} M$ be nonsingular. Then $M$ is retractable if and only if (I) holds. 
When we go to $e$-retractable modules, then, even for a nonsingular ${ }_{R} M$, we get a weakened version of property (I).

Recall that a submodule $C$ of $M$ is said to be a complement submodule of $M$ if $C$ has no proper essential extension in $M$. When ${ }_{R} M$ is nonsingular, then, to any submodule $U$ of $M$, there corresponds a unique complement, $U^{e}$, in $M$, such that $U<{ }_{R} U^{e}[3$, p.61].

THEOREM 2.4. Let ${ }_{R} M$ be nonsingular. Then the following are equivalent:

(i) $M$ is e-retractable .

(ii) For any complement $C$ in $M, S_{M} I_{B}(C)<{ }_{R}^{\prime} C$.

(iii) If $U<{ }_{R} V<{ }_{R} M$ and $V$ is a complement in $M$, then:

$$
I_{B}(U)<_{B}^{\prime}(V) \Rightarrow U<_{R}^{\prime} V .
$$

Proof: (i) $\Rightarrow$ (ii): Assume that $M$ is e-retractable, and let $0 \neq C$ be a complement in $M$. Let $0 \neq x \in C$ and set $Y=(R x)^{e}$. Since $Y \subseteq C$, we have $I_{B}(Y) \subseteq I_{B}(C)$, and since $0 \neq Y$ is a complement in $M$ and $M$ is e-retractable, there is $0 \neq b \in I_{B}(Y)$. Then $M b \subseteq Y$ and, since $R x<{ }_{R}^{\prime} Y$, there is $0 \neq z \in M b \cap R x$. Therefore, $0 \neq z \in M I_{B}(C) \cap R x$, proving that $M I_{B}(C)<{ }_{R} C$. For $C=0$, (ii) is trivially true. (ii) $\Rightarrow$ (i) is clear.

(i) $\Rightarrow$ (iii): Assume (i) and let $U<{ }_{R} V$, where $V$ is a complement; suppose that $I_{B}(U)<^{\prime} I_{B}(V)$. If $V=0$, (iii) is trivially true. Thus assume $V \neq 0$ and let $0 \neq v \in V$. Then, by (i), $I_{B}\left[(R v)^{e}\right] \neq 0$, hence there is $0 \neq b \in I_{B}\left[(R v)^{e}\right] \cap I_{B}(U)$. Then, $0 \neq M b \subseteq(R v)^{e} \cap U$, and this implies, since $R v<^{\prime}(R v)^{e}$, that $0 \neq R v \cap M b \subseteq$ $R v \cap(R v)^{e} \cap U=R v \cap U$; therefore $U<_{R}^{\prime} V$.

(iii) $\Rightarrow$ (ii): Assume (iii) and let $V$ be any nonzero complement in $M$. From $I_{B}(V)=I_{B} S_{M} I_{B}(V)$ we have, in particular, $I_{B} S_{M} I_{B}(V)<^{\prime} I_{B}(V)$, which, by (iii) implies that $S_{M} I_{B}(V)<{ }_{R}^{\prime} V$.

As regards property (II), here again we find that it holds when $M$ is nondegenerate, whereas for $M$ nonsingular and retractable, (II) holds if and only if we have $H<'$ $I_{B}(M H)$ for each $H<{ }_{B} B$. This last will follow as a Corollary from the next Theorem, which gives the relationship between properties (I) and (II).

THEOREM 2.5 .

(a) Given (I), then (II) holds if and only if $B<^{\prime} I_{B} S_{M}(B)$ for each $H<{ }_{B} B$.

(b) Given (II), then (I) holds if and only if $S_{M} I_{B}(U)<{ }_{R} U$ for each $U<{ }_{R} M$.

Proof: (a) Let (I) be given. Suppose that (II) holds and let $H<{ }_{B} B$. Then $S_{M} I_{B} S_{M}(H)=S_{M}(H)$ implies in particular that $S_{M}(H)<^{\prime} S_{M} I_{B} S_{M}(H)$, and this last, combined with $H<I_{B} S_{M}(H)$, implies, by (II), that $H<I_{B} S_{M}(H)$. 
Conversely, assume that $H<I_{B} S_{M}(H)$ for $H<{ }_{B} B$. To prove (II), assume first that $B<{ }_{B}^{\prime} J$. Then we have: $S_{M}(H)<S_{M}(J), H<I_{B} S_{M}(H)<I_{B} S_{M}(J)$ and $H<{ }_{B}^{\prime} J<^{\prime} I_{B} S_{M}(J)$; therefore, $H<^{\prime} I_{B} S_{M}(J)$, which implies that $I_{B} S_{M}(H)<^{\prime}$ $I_{B} S_{M}(J)$, and this last gives, by (I), that $S_{M}(H)<S_{M}(J)$. For the other direction of (II), assume that $S_{M}(B)<{ }^{\prime} S_{M}(J)$, where $H<{ }_{B} J<{ }_{B} B$; then, using (I), we have: $H<^{\prime} I_{B} S_{M}(H)<^{\prime} I_{B} S_{M}(J)$, hence $H<^{\prime} I_{B} S_{M}(J)$. But $H<{ }_{B} J<I_{B} S_{M}(J)$, hence $H<{ }_{B}^{\prime} J$.

(b) Let (II) be given. If (I) holds, then we know, by Proposition 2.1, that $S_{M} I_{B}(U)<{ }_{R}^{\prime} U$ for each $U<{ }_{R} M$. Conversely, assume that $S_{M} I_{B}(U)<{ }_{R}^{\prime} U$ for each $U<{ }_{R} M$. To prove (I), assume first that $U<{ }_{R} V$. Then, we have: $I_{B}(U)<I_{B}(V)$, $S_{M} I_{B}(U)<S_{M} I_{B}(V)<{ }_{R}^{\prime} V$ and $S_{M} I_{B}(U)<{ }_{R}^{\prime} U<{ }_{R}^{\prime} V$; therefore, $S_{M} I_{B}(U)<{ }_{R}^{\prime} V$ and hence $S_{M} I_{B}(U)<S_{M} I_{B}(V)$, which, by (II), implies that $I_{B}(U)<1 I_{B}(V)$. For the other direction of (I), assume that $I_{B}(U)<I_{B}(V)$, with $U<{ }_{R} V<{ }_{R} M$. Then, using (II), we have $S_{M} I_{B}(U)<^{\prime} S_{M} I_{B}(V)<_{R}^{\prime} V$, hence $S_{M} I_{B}(U)<{ }_{R}^{\prime} V$. But $S_{M} I_{B}(U)<{ }_{R}^{\prime} U<{ }_{R} V$, hence $U<{ }_{R}^{\prime} V$.

COROLLARY 2.6. Let ${ }_{R} M$ be nonsingular and retractable. Then (II) holds if and only if $H<I_{B} S_{M}(H)$ for each $H<{ }_{B} B$.

As mentioned at the beginning of this section, nondegenerate modules are one example of modules satisfying both of properties (I) and (II). Another example is that of a finitely generated quasi-projective nonsingular retractable module, for it is known that, when ${ }_{R} M$ is finitely generated quasi-projective, we have $H=I_{B} S_{M}(H)$ for each $H<{ }_{B} B$ (see for example [1, Proposition 4.9]).

Another way to see the difference between retractable and nondegenerate modules is to note that, for any $U<{ }_{R} M, T U<S_{M} I_{B}(U)<U$, and to recall that $M$ is nondegenerate if and only if $T U<{ }_{R}^{\prime} U$ for every $U<{ }_{R} M$ [6, Proposition 3.1]. It follows that $M$ is nondegenerate if and only if $S_{M} I_{B}(U)<{ }_{R}^{\prime} U$ and $T U<S_{M} I_{B}(U)$, for each $U<{ }_{R} M$, that is, if and only if $M$ is retractable and $T U<S_{M} I_{B}(U)$ for each $U<{ }_{R} M$ (using Proposition 2.1). For $M$ nonsingular, the condition $T U<{ }^{\prime} S_{M} I_{B}(U)$ for each $U<{ }_{R} M$ is equivalent to $T M<_{R}^{\prime} M$; more generally, we have:

THEOREM 2.7.

(i) For any ${ }_{R} M, T M<{ }_{R}^{\prime} M \Rightarrow T N<{ }^{\prime} M \operatorname{Hom}_{R}(M, N)$ for every nonsingular ${ }_{R} N$; if $M$ is nonsingular, the converse holds also.

(ii) $T<{ }_{R}^{\prime} R \Rightarrow T N<{ }_{R}^{\prime} N$ for every nonsingular $N$; if $R$ is left nonsingular, the converse holds also.

Proof: (i) Assume that $T M<{ }_{R}^{\prime} M$, and let ${ }_{R} N$ be nonsingular. Let $0 \neq$ $n \in M \operatorname{Hom}_{R}(M, N)$ and write $n=\sum_{i=1}^{k} m_{i} f_{i}$, with $0 \neq m_{i} \in M$ and $0 \neq f_{i} \in$ 
$\operatorname{Hom}_{R}(M, N)$, (assume also $m_{i} f_{i} \neq 0$ ), for $i=1, \ldots, k$. Set $K_{i}=\left\{r \in R: r m_{i} \in\right.$ $T M\}$; then $T M<{ }_{R}^{\prime} M$ implies that $K_{i}<{ }_{R} R$; hence, since $0 \neq m_{i} f_{i} \in N$ and $N$ is nonsingular, we have $0 \neq K_{i} m_{i} f_{i} \subseteq T M f_{i} \cap R m_{i} f_{i}$, for $i=1, \ldots, k$. Let $J=l_{R}(n)=$ $\{r \in R: r n=0\}$; then, since $N$ is nonsingular and $n \neq 0, J$ is not an essential left ideal of $R$. Let $0 \neq L$ be a left ideal of $R$ such that $L \cap J=0$. Since $\bigcap_{i=1}^{k} K_{i}<{ }_{R}^{\prime} R$, there is $0 \neq s \in L \cap\left(\bigcap_{i=1}^{k} K_{i}\right)$. We have: $0 \neq s n=s\left(\sum_{i=1}^{k} m_{i} f_{i}\right)=\sum_{i=1}^{k} s m_{i} f_{i} \in \sum_{i=1}^{k} T M f_{i}$, since $s \in \bigcap_{i=1}^{k} K_{i}$. Therefore, since $M f_{i} \subseteq N, 0 \neq s n \in T N \cap R n$, which shows that $T N<^{\prime} M \operatorname{Hom}_{R}(M, N)$. The second statement is clear.

(ii) Assume that $T<{ }_{R} R$ and let ${ }_{R} N$ be nonsingular. Let $0 \neq n \in N$; then $l_{R}(n)=\{r \in R: r n=0\}$ is not essential as a left ideal of $R$, so there is a nonzero left ideal $J$ in $R$ such that $J \cap l_{R}(n)=0$. Then $J \cap T \neq 0$ and $0 \neq(J \cap T) n \subseteq J n \cap T N$; so $T N<{ }_{R}^{\prime} N$. Again, the second statement is clear.

Corollary 2.8. Let ${ }_{R} M$ be nonsingular. Then $T M<{ }_{R} M$ if and only if $T U<^{\prime} S_{M} I_{B}(U)$ for every $U<{ }_{R} M$; and $M$ is nondegenerate if and only if $T M<^{\prime}$ ${ }_{R} M$ and $M$ is retractable.

\section{ENDOMORPHISM RINGS OF NONSINGULAR RETRACTABLE MODULES}

As mentioned in the Introduction, our aim is to generalise the result:

( $N$ ) Let ${ }_{R} M$ be nonsingular and nondegenerate. Then $B=\operatorname{End}_{R} M$ is a left $C S$ ring if and only if $M$ is a $C S$ module [6, Corollary 3.11],

from nondegenerate to retractable modules.

In [6], this result followed easily from the fact that, for a nonsingular nondegenerate ${ }_{R} M$, there is a projectivity (that is, an order-preserving bijection) between the complement submodules of $M$ and the complement left ideals of $B$, given by $U \rightarrow I_{B}(U)$, for $U$ a complement of $M$, and $H \rightarrow\left[S_{M}(H)\right]^{e}$, for $H$ a left complement of $B$. For a nonsingular retractable $M$, however, the maps $U \rightarrow I_{B}(U)$ and $H \rightarrow\left[S_{M}(H)\right]^{e}$ will not give us a projectivity between the complements of $M$ and the left complements of $B$ unless we have the additional condition $H<I_{B} S_{M}(H)$ for every $B<{ }_{B} B$, as we now show.

Set $\mathcal{C}^{e}=\left\{U<{ }_{R} M: U\right.$ is a complement submodule of $\left.M\right\}$, and $\mathcal{C}^{1}(B)=\{H<$ ${ }_{B} B: H$ is a complement left ideal of $\left.B\right\}$.

THEOREM 3.1. Let ${ }_{R} M$ be nonsingular and retractable. Then the maps $U \rightarrow$ $I_{B}(U)$ and $H \rightarrow\left[S_{M}(H)\right]^{e}$ determine a projectivity between $\mathcal{C}^{e}$ and $\mathcal{C}^{1}(B)$ if and only if $K<^{\prime} I_{B} S_{M}(K)$ for every $K<{ }_{B} B$. 
Proof: Assume that $K<^{\prime} I_{B} S_{M}(K)$ for every $K<{ }_{B} B$. Then, by Corollary 2.6, property (II) holds; also, by Theorem 2.2, property (I) holds. Let $U \in \mathcal{C}^{e}$ and suppose that $I_{B}(U)<{ }_{B}^{\prime} J$. By Zorn's Lemma, we may assume that $J \in \mathcal{C}^{1}(B)$. Since (II) holds, $I_{B}(U)<{ }_{B}^{\prime} J$ implies that $S_{M} I_{B}(U)<S_{M}(J)$; since $M$ is retractable, $S_{M} I_{B}(U)<{ }_{R}^{\prime} U$, by Proposition 2.1. Therefore, $U=\left[S_{M} I_{B}(U)\right]^{e}=\left[S_{M}(J)\right]^{e}$, so that $S_{M}(J) \subseteq U$ and hence $J \subseteq I_{B}(U)$; then $I_{B}(U)=J$, that is $I_{B}$ maps $U \in \mathcal{C}^{e}$ to $I_{B}(U) \in \mathcal{C}^{1}(B)$. Clearly, for any $H<{ }_{B} B,\left[S_{M}(H)\right]^{e} \in \mathcal{C}^{e}$.

Let $H \in \mathcal{C}^{1}(B)$; by (I), $S_{M}(H)<^{\prime}\left[S_{M}(H)\right]^{e}$ - implies that $I_{B} S_{M}(H)<^{\prime}$ $I_{B}\left\{\left[S_{M}(H)\right]^{e}\right\}$. Then $H<_{B}^{\prime} I_{B} S_{M}(H)<^{\prime} I_{B}\left\{\left[S_{M}(H)\right]^{e}\right\}$ implies that $H=I_{B} S_{M}(H)=I_{B}\left\{\left[S_{M}(H)\right]^{e}\right\}$.

We have: $U \in \mathcal{C}^{e} \rightarrow I_{B}(U) \in \mathcal{C}^{1}(B) \rightarrow\left[S_{M} I_{B}(U)\right]^{e}=U$, and $H \in \mathcal{C}^{1}(B) \rightarrow$ $\left[S_{M}(H)\right]^{e} \in \mathcal{C}^{e} \rightarrow I_{B}\left\{\left[S_{M}(H)\right]^{e}\right\}=H$.

Hence the two order-preserving maps are inverses of each other and so determine a projectivity between $\mathcal{C}^{e}$ and $\mathcal{C}^{1}(B)$.

Conversely, assume that the maps $U \rightarrow I_{B}(U)$ and $H \rightarrow\left[S_{M}(H)\right]^{e}$ determine a projectivity between $\mathcal{C}^{e}$ and $\mathcal{C}^{1}(B)$. Then, if $H \in \mathcal{C}^{1}(B), H=I_{B}\left\{\left[S_{M}(H)\right]^{e}\right\}$; hence, since $H<I_{B} S_{M}(H)<I_{B}\left\{\left[S_{M}(H)\right]^{e}\right\}$, it follows that $H=I_{B} S_{M}(H)$. Let $K$ be any left ideal in $B$; there is $J \in \mathcal{C}^{1}(B)$ such that $K<_{B}^{\prime} J$. We have $J=I_{B} S_{M}(J)$, $S_{M}(K)<S_{M}(J)$, and $K<I_{B} S_{M}(K)<I_{B} S_{M}(J)=J$; so $K<{ }_{B}^{\prime} J$ implies that $K<^{\prime} I_{B} S_{M}(K)$, and the proof is complete.

In view of Theorem 3.1 when $M$ is nonsingular and retractable, we need to find a way other than the projectivity between complements, in order to transfer the $C S$ property between $M$ and $\operatorname{End}_{R} M$. Our proof will make use of the injective hull, $\widetilde{M}$, of $M$ and its endomorphism ring, $A=\operatorname{End}_{R} \widetilde{M}$. Notation: Let $r_{B}(U)=\{b \in B$ : $U b=0\}$, for $U<{ }_{R} M$.

THEOREM 3.2. Let ${ }_{R} M$ be nonsingular and retractable. Then $B=\operatorname{End}_{R} M$ is a left $C S$ ring if and only if $M$ is a $C S$ module.

Proof: Let $M$ be nonsingular, retractable and $C S$. Since $M$ is nonsingular and retractable, we have, by [5, Theorem 3.1] that $B$ is left nonsingular, $B<{ }_{B} A$ and $A$ is the maximal left quotient ring of $B$. Since $M$ is a $C S$ module, if $U \in \mathcal{C}^{e}$ and $U \neq M$, then we have $U=M e$, for $1 \neq e=e^{2} \in B$, and therefore $0 \neq 1-e \in r_{B}(U)$. Since $M$ is nonsingular, we know by [4, Theorem 3.5] that, if for every complement $U$ in $M$ such that $U \neq M$ we have $r_{B}(U) \neq 0$, then $B$ has nonzero intersection with every nonzero right ideal of $A=\operatorname{End}_{R} \widetilde{M}$. Hence, the left nonsingular ring $B$ has nonzero intersection with every nonzero right ideal of its maximal left quotient ring $A$; therefore, it follows by Utumi's Theorem [7, Theorem 2.2] that every complement left ideal in $B$ is a left annihilator in $B$. But, by [6, Theorem 3.13], since $M$ is nonsingular 
and $C S$, every left annihilator in $B$ is a direct summand in $B$ (that is, $B$ is a Baer ring). Hence every complement left ideal in $B$ is a direct summand in $B$, and $B$ is a left $C S$ ring.

As mentioned in the Introduction, the converse, that is, for ${ }_{R} M$ nonsingular retractable $B$ left $C S$ implies $M C S$, is proved in [2, Theorem 3.1]. However, for completeness, we give a (slightly shorter version of the) proof which makes use of Theorem 2.4. Let $M$ be nonsingular and $e$-retractable and assume that $B$ is a left $C S$ ring. Let $U \in \mathcal{C}^{e}$ and set $H=I_{B}(U)$. There is $K \in \mathcal{C}^{1}(B)$ such that $B<{ }_{B}^{\prime} K$, and, since $B$ is a left $C S$ ring, $K=B e$ for $e=e^{2} \in B$. We have $S_{M}(H)<S_{M}(K)=S_{M}(B e)=M e$, and $I_{B} S_{M}(K)=I_{B}(M e)$. Clearly, $e \in I_{B}(M e)$, so that $B e \subseteq I_{B}(M e)$; on the other hand, if $b \in I_{B}(M e)$, then, for any $m \in M, m b=m_{1} e$ for some $m_{1} \in M$, hence $m b e=m_{1} e^{2}=m_{1} e=m b$, that is, $b=b e \in B e$. Therefore $I_{B}(M e)=B e=K$, that is, $I_{B} S_{M}(K)=K$; hence, since $H<{ }_{B}^{\prime} K$, we have $H<I_{B} S_{M}(H)<I_{B} S_{M}(K)=K$. Thus, we have $S_{M}(H)<S_{M}(K)$ and $I_{B} S_{M}(H)<^{\prime} I_{B} S_{M}(K)$, with $S_{M}(K)=M e$ a direct summand and hence a complement in $M$. Therefore, by Theorem 2.4, it follows that $S_{M}(H)<^{\prime} S_{M}(K)$. Since $M$ is $e$-retractable and $U \in \mathcal{C}^{e}$, we have, again by Theorem 2.4, $S_{M} I_{B}(U)<{ }_{R}^{\prime} U$. Therefore, we have $U=\left[S_{M} I_{B}(U)\right]^{e}=\left[S_{M}(H)\right]^{e}=S_{M}(K)$; that is, $U=S_{M}(K)=M e$, and $U$ is a direct summand in $M$, proving that $M$ is a $C S$ module.

REMARK. Note that the first half of the proof of Theorem 3.2 does not go through if we weaken the hypothesis to " $M$ nonsingular $e$-retractable" because we need retractability to show that $B$ is essential as a left $B$-submodule of $A$, in order to have $A=\operatorname{End}_{R} \widetilde{M}$ equal to the maximal left quotient ring of $A$. The weaker hypothesis of $e$-retractability is, for $M$ nonsingular, equivalent to the weaker result: $B$ has nonzero intersection with every nonzero left ideal of $A[4$, Theorem 3.4].

\section{REFERENCES}

[1] T. Albu and C. Nastasescu, Relative finiteness in module theory (Dekker, New York, 1984).

[2] A.W. Chatters and S.M. Khuri, 'Endomorphism rings of modules over nonsingular CS rings', J. London Math. Soc. 21 (1980), 434-444.

[3] C. Faith, Lectures on injective modules and quotient rings 246, Lecture Notes in Mathematics (Springer-Verlag, Heidelberg, Berlin, New York, 1972).

[4] S.M. Khuri, 'Baer endomorphism rings and closure operators', Canad. J. Math. 30 (1978), 1070-1078.

[5] S.M. Khuri, 'Endomorphism rings of nonsingular modules', Ann. Sci. Math. Quebec 4 (1980), 145-152.

[6] S.M. Khuri, 'Correspondence theorems for modules and their endomorphism rings', $J$. Algebra 122 (1989), 380-396: 
[7] Y. Utumi, 'On rings of which any one-sided quotient ring is two-sided', Proc. Amer. Math. Soc. 14 (1963), 141-147.

Department of Mathematics

East Carolina University

Greenville, North Carolina 27858

United States of America 